\title{
Towards an Identification of Tumor Growth Parameters from Time Series of Images ${ }^{\star}$
}

\author{
Ender Konukoglu ${ }^{1}$, Olivier Clatz ${ }^{1,3}$, Pierre-Yves Bondiau ${ }^{2}$, \\ Maxime Sermesant ${ }^{1}$, Hervé Delingette ${ }^{1}$, and Nicholas Ayache ${ }^{1}$ \\ ${ }^{1}$ Asclepios Research Project, INRIA Sophia Antipolis, France \\ ender.konukoglu@sophia.inria.fr \\ ${ }^{2}$ Centre Antoine Lacassagne, Nice, France \\ ${ }^{3}$ SPL Harvard, Boston, USA
}

\begin{abstract}
In cancer treatment, understanding the aggressiveness of the tumor is essential in therapy planning and patient follow-up. In this article, we present a novel method for quantifying the speed of invasion of gliomas in white and grey matter from time series of magnetic resonance (MR) images. The proposed approach is based on mathematical tumor growth models using the reaction-diffusion formalism. The quantification process is formulated by an inverse problem and solved using anisotropic fast marching method yielding an efficient algorithm. It is tested on a few images to get a first proof of concept with promising new results.
\end{abstract}

\section{Introduction}

Glial based tumors account for approximately $40-45 \%$ of all primary intracranial cancer, forming the largest class in this pathology [1]. Tumors within this group show a high variation extending from benign to fatal. Determining different characteristics of each specific case and following their change during the treatment is crucial in therapy planning and patient follow-up. Although medical imaging is not the sole source of information used for this, it plays an important role in understanding the pattern and speed of invasion of healthy tissue by cancerous cells. One of the most important hints that can be obtained from images is the progression of the Critical Target Volume (CTV) and Gross Tumor Volume (GTV), which are important in radiotherapy. For low grade gliomas, CTV corresponds to the enhanced region in the T2-weighted magnetic resonance images (MRI) and at its extent tumor cells are diffused into the brain tissue. In the case of high grade gliomas CTV corresponds to the extents of the tumor infiltrated edema 2. GTV in both cases is taken as the image abnormality in T1 weighted images corresponding to the region where tumor cells are dense. As shown by Giese et al., speed of invasion of tumor cells in grey and white matter are different, hence the speed of CTV progression [3]. Quantifying this progression of

\footnotetext{
* This work has been partly supported by the European Health-e-Child project (IST-2004-027749) and by the CompuTumor project (http://www-sop.inria.fr/ asclepios/projects/boston/).
} 
CTV in different tissues would be helpful in initial grading of the tumor and assessing the efficacy of the current treatment procedure.

Mathematical modeling of tumor growth dynamics gives us an insight on the physiology of the process by linking different types of observations under theoretical frameworks. There has been a large amount of models proposed to describe the growth dynamics of glial tumors. Different approaches can be coarsely classified into two groups, macroscopic and microscopic ones. Macroscopic models describe the evolution of local tumor cell densities and try to capture the dynamics by general equations 4 456. Most of the macroscopic models are based on the reaction-diffusion formalism introduced by Murray in 74. .

In this paper we are proposing a method for quantifying the progression of the CTV of glial based tumors. The formulation is based on the tumor growth model proposed by Clatz et al. in [5], which uses reaction-diffusion formalism. With the proposed method, we obtain quantitative estimates for the speed of invasion in white and grey matter by solving the patient specific parameter identification problem for this growth model using MR images taken at two different time instances from the same patient. The parameter identification problem is formulated using the front approximation of reaction-diffusion equations, which results in anisotropic Eikonal equations. The anisotropic fast marching method proposed in 8 ] is used for numerical solutions yielding an efficient algorithm.

\section{Method}

Quantitative measures for the speed of CTV progression can be obtained using reaction-diffusion based growth models, which explain the invasion through diffusion process. Clatz et al. proposed such a model in the form of a FisherKolmogorov (F-KPP) equation in [5] based on the observation of Giese et al.:

$$
\frac{\partial u}{\partial t}=\nabla \cdot(\mathrm{D}(\mathbf{x}) \nabla u)+\rho u(1-u), \mathrm{D}(\mathbf{x}) \nabla u \cdot \vec{n}_{\Sigma}=0
$$

where $u$ can be seen as the normalized tumor cell density or the probability of finding a tumor at a given point, $\mathrm{D}$ is the diffusion tensor explaining the invasion of tumor cells, $\rho$ is the proliferation rate and $\vec{n}_{\Sigma}$ corresponds to the normal vector of the brain surface. We focus in this article on the D matrix, which defines anisotropic diffusion on the white matter following the main fiber directions and isotropic diffusion on the grey matter, constructed as:

$$
\mathrm{D}(\mathbf{x})= \begin{cases}d_{g} \mathbf{I} & \text { if } \mathbf{x} \text { is in grey matter } \\ d_{w} \mathrm{D}_{\mathrm{w}}(\mathbf{x}) & \text { if } \mathbf{x} \text { is in white matter }\end{cases}
$$

where $\mathrm{D}_{\mathrm{w}}$ is the water diffusion tensor obtained from MR diffusion tensor imaging (MR-DTI) providing the fiber direction. Quantities explaining the speed of invasion are $d_{g}$ and $d_{w}$, diffusion coefficients in grey and white matter respectively. Identification of these parameters for each patient using images taken at two different times corresponds to the identification process. 


\subsection{Front Approximation of Reaction-Diffusion Equations}

The model given in Equation 1 requires tumor cell density $u$ to be known at every point as an initial condition. However, this is not the case for medical images where only contours around GTV and CTV are available. The front motion approximation of reaction-diffusion equations offers a solution for this discrepancy between information needed and observations available [8. These approximations formulate the motion of the tumor front (the visible contour) based on a reaction-diffusion equation such as the one given in Equation 1 . Taking the contour around the CTV as the last visible tumor front we can use such an approximation to model its evolution.

The front motion approximation for reaction-diffusion equations is based on the fact that these PDEs have travelling wave solutions under certain conditions. This can be illustrated in the simple one dimensional F-KPP equation where $D$ is scalar. In 9 it is shown that any initial condition with compact support will evolve and converge to a traveling wave of the form $u=u(x-c t)$ in time, where $c=2 \sqrt{D \rho}$ is the asymptotic speed of a point taken on the front. The convergence of the front and the speed of the point $u=0.5$ can be seen in Figure 1. The convergence property is carried to higher dimensions for contours
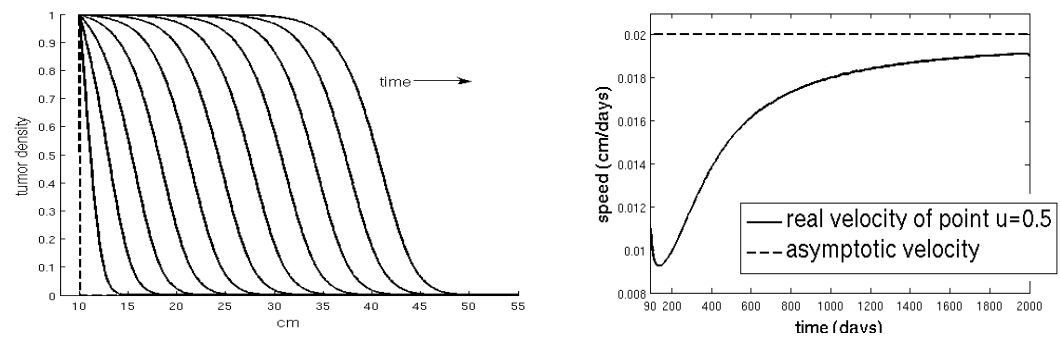

Fig. 1. Left: Front evolution starting from a step function. Steady wave-front is translating in time. Right: Speed of wave-front plotted versus time showing the convergence behavior and the asymptotic speed.

on the travelling wave with the asymptotic speed $c=2 \sqrt{\rho \mathbf{n}^{t} \mathrm{Dn}}$ under the condition that coefficients of the equation are constant and the front is planar, meaning the front has no curvature and its normal is given by $\mathbf{n}$. For more general fronts $\Gamma$ like the tumor front mentioned where curvature exists and coefficients vary spatially we make the assumption that required conditions are satisfied within a voxel. Based on this assumption we can simply derive the travelling time formulation for $\Gamma$ :

$$
\sqrt{\nabla T^{t} \mathrm{D} \nabla T}=\frac{1}{2 \sqrt{\rho}}
$$

where $T(\mathbf{x})$ represents the time at which $\Gamma$ passes from point $\mathbf{x}[10$. 


\subsection{Numerical Method}

The travelling time formulation given in Equation 3 is an anisotropic Eikonal equation. Very efficient methods for isotropic Eikonal equation $F|\nabla T|=1$ have been proposed like fast marching (FM) methods [11. These methods are based on the fact that characteristic directions of the equation are parallel to $\nabla T$ [12. However, the anisotropic case poses extra difficulties for efficient numerical algorithms because this property is not satisfied [12. The method we used to solve Equation 3 numerically is based on the original fast marching idea with the addition of a recursive correction phase to compensate the effects of anisotropy. In [8], it is demonstrated that this algorithm is fast and efficient in the case of high anisotropies and general meshes. Having an efficient numerical method for Equation 3 is essential in solving the parameter identification problem explained in the next section.

\subsection{Parameter Identification Problem}

The parameter identification process uses the front motion approximation given in Equation 3 In order to formulate this inverse problem we follow the modeling assumption as given in [5] and state $T\left(\Gamma_{1}\right)=0$ and $T\left(\Gamma_{2}\right)=t_{2}-t_{1}$, where $\Gamma_{1}$ and $\Gamma_{2}$ corresponds to contours around CTV regions observed in images taken at time $t_{1}$ and $t_{2}$ respectively. Parameter identification process tries to find parameters $d_{g}$ and $d_{w}$ that create a $T$ function that would satisfy these conditions. Notice that $\rho$ is a multiplicative factor in the travelling time formulation and it cannot be determined independently from the $D$ matrix by just looking at the motion of the tumor front. To tackle this, we treat $\rho$ as a known constant in the parameter identification problem. $t_{1}$ is not available in clinical circumstances hence we use $t_{2}-t_{1}$. The formulation can be given as the minimization problem

$$
\begin{aligned}
C\left(d_{w}, d_{g}\right) & =\frac{1}{2}\left(\operatorname{dist}\left(\Gamma_{2}, \widehat{\Gamma_{2}}\right)+\operatorname{dist}\left(\widehat{\Gamma_{2}}, \Gamma_{2}\right)\right), d_{w}>0 \text { and } d_{g}>0 \\
\widehat{\Gamma_{2}} & =\left\{\mathbf{x} \mid T(\mathbf{x})=t_{2}-t_{1}, \sqrt{\nabla T^{t} \mathrm{D} \nabla T}=\frac{1}{2 \sqrt{\rho}}, T\left(\Gamma_{1}\right)=0\right\}
\end{aligned}
$$

where $C$ is the objective function to minimize with respect to $d_{w}$ and $d_{g}, \widehat{\Gamma_{2}}$ is the computed contour using the front approximation with the given parameters and $\operatorname{dist}(A, B)$ is the distance between two isosurfaces taken as mean distance from voxels of $\mathrm{A}$ to the closest voxel of $\mathrm{B}$.

The minimizing parameters $d_{w}^{*}$ and $d_{g}^{*}$ of the function $C$ will create the closest contour ${\widehat{\Gamma_{2}}}^{*}$ to the observed contour $\Gamma_{2}$. This formulation poses a multidimensional minimization problem for which several methods have been proposed. One crucial observation is that explicit derivatives of $C$ with respect to the variables are not available. Although we have constraints on $d_{w}$ and $d_{g}$, these are not very restrictive so we choose to use the unconstrained minimization algorithm proposed by Powell in [13 and restrict the domain computation. The attractive feature of this algorithm is that it does not require derivatives of the objective 
function. Instead, local quadratic approximations of the objective functions are obtained and used in the minimization. The algorithm requires instances of the objective function to construct the quadratic approximation - which is computed using anisotropic fast marching - and updates it as the minimization proceeds. This minimization algorithm is prone to getting stuck at local minima. However, we observed in our experiments that the minimization surface is convex hence, this does not pose a problem.

$$
\begin{aligned}
& t_{1}-t_{2}=120 \text { days } \\
& d_{w}^{*}=4.7 \mathrm{~mm}^{2} / \text { day } \\
& d_{g}^{*}=4.3 \times 10^{-2} \mathrm{~mm}^{2} / \text { day }
\end{aligned}
$$

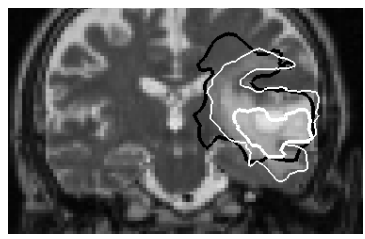

$t_{1}-t_{2}=270$ days

$d_{w}^{*}=0.5 \mathrm{~mm}^{2} /$ day

$d_{g}^{*}=2.7 \times 10^{-3} \mathrm{~mm}^{2} /$ day
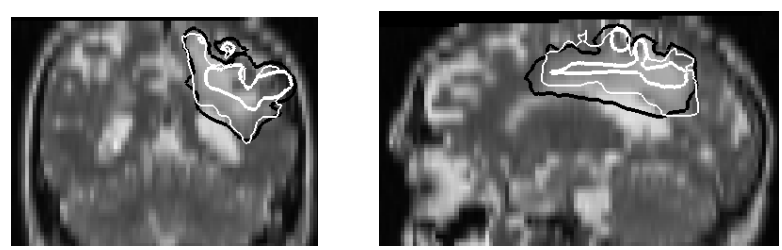

(b)

(a)
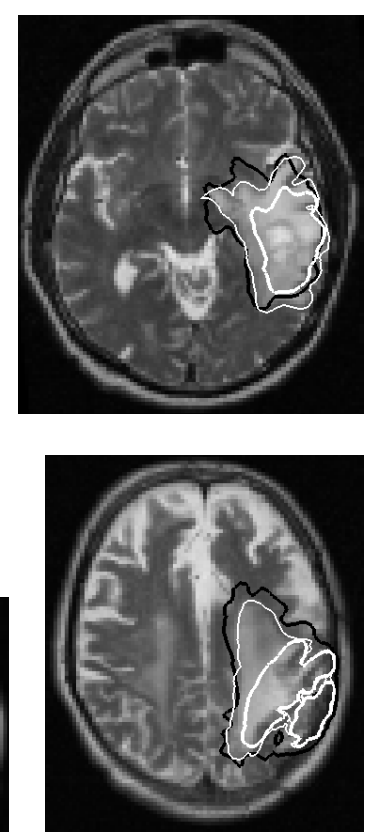

Fig. 2. Minimizing tumor front computed in the identification problem $\widehat{\Gamma}_{2}^{*}$ (thick black contours) is plotted with the initial and the final tumor fronts manually segmented from the images at taken at $t_{1}$ and $t_{2}$ respectively, $\left(\Gamma_{1}\right.$ - thick white contours, $\Gamma_{2}$ - thin white contours). Underlying images are T2 weighted images taken at the second time instance. Estimated growth parameters (appearent diffusion coefficients) $d_{g}^{*}$ and $d_{w}^{*}$ for these patients are also given.

\section{$3 \quad$ Results}

We illustrate the use of the proposed algorithm on two different patient images both containing high grade gliomas, glioblastoma multiforme. In order to solve the parameter identification problem on patient images we need to construct tumor diffusion tensors as given in Equation 2, which requires MR-DTI and white matter segmentation of the patient. Diffusion images are not available for every patient and existence of the tumor and the low quality of patient images make it impossible to obtain an accurate white matter segmentation. Thus, in 
our experiments we used data acquired from a healthy subject, which consists of T1 and T2 weighted images alongside MR-DTI. This data is registered to the patient space using global affine transformation found using T1 weighted images. Tensors in the MR-DTI were re-oriented using finite strain strategy after applying the global affine transformation to take into account the effects of the transformation on the tensors 14. To obtain the white matter segmentation, we use fractional anisotropy (FA) values of the DTI data and let points with $\mathrm{FA}>0.3$ form the white matter as an arbitrary value chosen for illustration of the method. This way we obtain perfect correspondence between diffusion tensors and the white matter segmentation. Lastly the $\rho$ is set to 0.012 /day as proposed in [4].
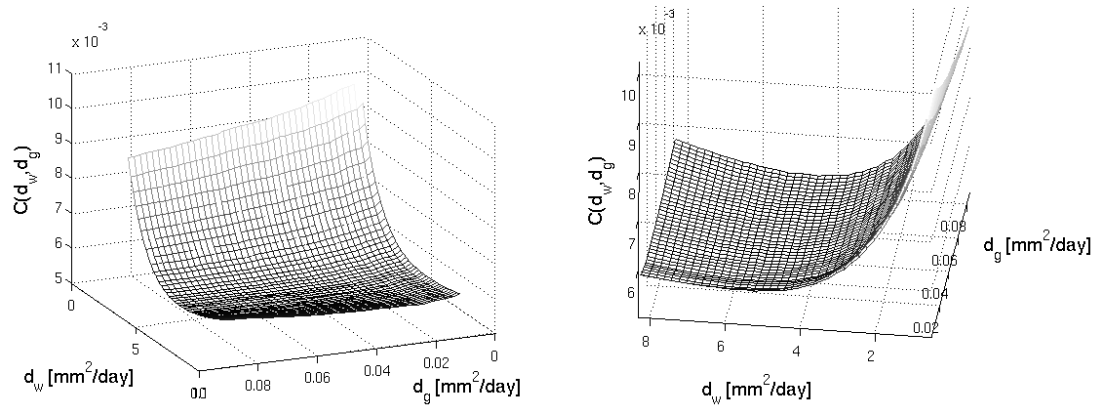

Fig. 3. The objective function $C\left(d_{w}, d_{g}\right)$ (minimization surface) for the patient whose image are given in Figure 2(a) is plotted from two different views

The minimization algorithm explained in Section 2.3 is applied to the patient images given in Figure 2(a) and Figure 2(b). Tumor segmentation at the initial time (thick white contour), tumor segmentation at the final time (thin white contour) the computed tumor front minimizing the objective function $\widehat{\Gamma}^{*}$ (thick black contour) are drawn on T2 weigthed images taken at the second time instance showing CTV at $t_{2}$. The minimizing parameters $d_{w}^{*}$ and $d_{g}^{*}$ for each patient are also given in these figures. In Figure 3 we plot the minimization surface (the function $C\left(d_{w}, d_{g}\right)$ ) computed for the patient image given in Figure 2(a). Notice the minimization surface has a wide valley which makes the optimization hard, on the other hand the surface is convex yielding only the global minimum. The minimizing criterion, which is the symmetric distance between the computed front and the delineated one, at this minimum reaches the value $0.05 \mathrm{~mm}$ for the first patient data and $0.078 \mathrm{~mm}$ for the second patient data demonstrating the similarity between the computed front and the real delineation.

\section{Discussion}

In this article, we proposed a novel method to quantify the speed of tumor invasion in white and grey matter for gliomas on MR images using mathematical 
growth models. This tool can be helpful in tumor grading and patient follow-up as it gives quantitative values about the growth of the tumor. Quantification process is formulated as a parameter identification problem and solved using the anisotropic fast marching method and multidimensional optimization. The speed estimates are given in terms of apparent diffusion coefficients $d_{g}$ and $d_{w}$, which are used to construct tumor diffusion tensor as suggested by the growth model given in Equation 1 .

Results given in Section 3 demonstrate the functioning of the proposed method. We observe that minimizing contours given in Figure 2 matches the actual Critical Target Volume delineation given in the image reasonably well. The discrepancies between these contours and the underlying CTVs are caused by different factors such as the use of registered DTI data instead of the one from the patient, lack of a good white matter segmentation of the patient, not taking into account the mass effect caused by the tumor and the fact that identification process assumes tumor growth is perfectly explained by the mathematical growth model used. The diffusion coefficients obtained, shows a big difference between the speed of the tumor in grey matter and in white matter coherent with the experimental results given in 3 .

The method has been demonstrated on high grade gliomas where CTV corresponds to the edema region. However, the same tool can also be applied, and will be future work, to low grade gliomas since reaction-diffusion growth models have been proposed for these types of tumors as well [6]. Clinical values of the estimated diffusion coefficients should be assessed using a large database in order to understand their importance. Finally introducing different imaging modalities can give us the opportunity to find parameters more accurately and identify more parameters such as $\rho$.

\section{References}

1. Tovi, M.: Mr imaging in cerebral gliomas analysis of tumour tissue components. Acta Radiol. Suppl. (1993)

2. Kantor, G., Loiseau, H., Vital, A., Mazeron, J.: Descriptions of gtv and ctv for radiation therapy of adult glioma. Cancer Radiother (2001)

3. Giese, A., Kluwe, L., Laube, B., Meissner, H., Berens, M., Westphal, M.: Migration of human glioma cells on myelin. Neurosurgery 38(4) (1996)

4. Swanson, K., Alvord, E., Murray, J.: Virtual brain tumours (gliomas) enhance the reality of medical imaging and highlight inadequacies of current therapy. British Journal of Cancer 86 (2002)

5. Clatz, O., Sermesant, M., Bondiau, P., Delingette, H., Warfield, S., Malandain, G., Ayache, N.: Realistic simulation of the $3 \mathrm{~d}$ growth of brain tumors in $\mathrm{mr}$ images coupling diffusion with biomechanical deformation. IEEE T.M.I. 24(10) (2005)

6. Jbabdi, S., Mandonnet, E., Duffau, H., Capelle, L., Swanson, K., Pélégrini-Issac, M., Guillevin, R., Benali, H.: Simulation of anisotropic growth of low-grade gliomas using diffusion tensor imaging. Magnetic Reson. in Med. 54 (2005)

7. Murray, J.: Mathematical Biology. Springer, Heidelberg (2002) 
8. Konukoglu, E., Sermesant, M., Clatz, O., Peyrat, J.M., Delingette, H., Ayache, N.: A recursive anisotropic fast marching approach to reaction diffusion equation: Application to tumor growth modeling. In: Karssemeijer, N., Lelieveldt, B. (eds.) IPMI 2007. LNCS, vol. 4584, Springer, Heidelberg (2007)

9. Aronson, D., Weinberger, H.: Multidimensional nonlinear diffusion arising in population genetics. Advances in Mathematics 30 (1978)

10. Keener, J., Sneyd, J.: Mathematical physiology. Springer, Heidelberg (1998)

11. Sethian, J.: Level set methods and fast marching methods: Evolving interfaces in computational geometry, fluid mechanics, computer vision, and materials science. Cambridge University Press, Cambridge (1999)

12. Sethian, J., Vladimirsky, A.: Ordered upwind methods for static hamilton-jacobi equations: theory and algorithms. SIAM J. Numer. Anal. 41 (2003)

13. Powell, M.: Uobyqa: unconstrained optimization by quadratic approximation. Math. Program. Ser. B 92 (2002)

14. Alexander, D., Pierpaoli, C., Basser, P., Gee, J.: Spatial transformations of diffusion tensor magnetic resonance images. IEEE Trans. Med. Imag. 20 (2001) 